\title{
Metáforas y Psicoanálisis Relacional
}

\author{
Gustavo Camino ${ }^{1}$ \\ Psicólogo Clinico, Community Mental Health Team - Coaling Island, Gibraltar
}

Una creciente concienciación social y demanda de psicología clínica en los servicios públicos para atender a los usuarios de salud mejor y en el menor tiempo posible, hace que los profesionales tengan que plantearse nuevas formas de trabajar. Sin embargo, el paradigma psicológico dominante que informa a los profesionales continúa siendo el cognitivo-conductual. El presente trabajo intenta responder a esta necesidad social proponiendo una serie de metáforas terapéuticas para utilizar en la práctica clínica, dándole un sentido y una base relacional, dentro de la psicoterapia intersubjetiva, focal y breve, que satisfacen también principios cognitivos y conductuales de cambio. Su justificación radica tanto en la naturaleza narrativa y pedagógica de las metáforas como en los potenciales efectos sumativos cuando se integra con técnicas y teorías relacionales.

Palabras clave: Metáforas, Psicoterapia intersubjetiva, focal y breve, integración, modelo cognitivo-conductual

A growing social awareness and demand of clinical psychology within public services to attend service users better and faster, are making professionals inclined to develop new ways of working. However, the psychological paradigm which continues to inform professionals is the cognitivebehavioural model. The present work atempts to respond to that social need by proposing a series of therapeutic metaphors to be used in the clinical practice, giving them a relational basis and meaning, within the brief, focal and intersubjective psychotherapy framework, but also satisfying cognitive-behavioural principles of change. The justification lies on the very narrative and educational nature of metaphors, as much as the potential summative effect that implies the integration with relational techniques and theories.

Key Words: metaphors, brief, focal and intersubjective psychotherapy, integration, cognitivebehavioural model.

English Title: Metaphors and Relational Psychoanalysis

Cita bibliográfica / Reference citation:

Camino, Gustavo (2019). Metáforas y psicoanálisis relacional. Clínica e Investigación Relacional, 13

(2): 500-509. [ISSN 1988-2939] [Recuperado de www.ceir.info ] DOI: 10.21110/19882939.2019.130211

\footnotetext{
1 Licenciado en psicología por la Universidad Hispalense en 1994. Tras licenciarme hasta mi marcha al Reino Unido (RU) en 2001, trabajé en ONGs andaluzas, como psicólogo. En RU comencé trabajando como profesor de psicología en colleges y posteriormente en 2003 pasé a ser psicólogo clínico adjunto, en el sistema nacional de salud (NHS) para completar mi especialidad y doctorado en Psicología Clínica en 2008 (Universidad de Hertfordshire). En mi periplo británico tuve ocasión de completar un máster en terapia cognitiva para la psicosis en 2013 (King's College) mientras trabajaba en servicios psiquiátricos hospitalarios. Posteriormente emigré a Gibraltar donde ejerzo en el sistema sanitario público (GHA) en un equipo de salud mental comunitario.
} 


\section{Introducción}

Muchos psicólogos clínicos defienden cada vez más a su profesión como un todo, más que por un enfoque exclusivo y definidor de su práctica profesional. El psicólogo clínico es un profesional que trabaja desde la perspectiva sanitaria y probablemente desde una institución pública, observando a su paciente de un modo holístico, y por tanto puede hacer uso de perspectivas diversas sin necesidad de caer en el fanatismo de un paradigma, en la dispersión o en un eclecticismo invertebrado. No obstante, y a pesar de esta tendencia integradora, no hay que perder de vista el origen y sentido de cada aspecto de la práctica clínica. En cualquier caso, no es por ello sorprendente que sea posible establecer una convergencia entre diversas corrientes teóricas y aplicadas, las cuales se dirigen cada vez más a trabajar en un contexto de tiempo limitado, enfocado a los intereses del paciente y con perspectivas donde la alianza terapéutica se considera un eslabón fundamental del cambio. De este modo, por ejemplo, desde la perspectiva cognitiva y psicoanalítica se están tratando de ofrecer abordajes breves y compatibles con sistemas sanitarios desbordados y costosos para las democracias occidentales. Tales corrientes convergentes pueden encontrar un origen en Estados Unidos, donde las dinámicas sociales e institucionales han podido ir generando dichos procesos desde bien entrado el siglo XX (i.e. Velasco-Fraile, 2009). Sin embargo, en nuestro país, así como en otros de similar estructura universitaria y asistencial, las perspectivas dinámicas siguen marginadas, en favor de la abrumadora presencia institucional de la terapia cognitivaconductual (sobre todo en su versión más clásica, que en sus siglas en inglés se conoce como CBT). Por ejemplo, en el Reino Unido existe un programa nacional llamado Improving Access to Psychological Therapies (IAPS) (NCCMH, 2019), en el que se administra CBT de manera masiva, en formatos de asistencia telefónica, fomentando la lectura de autoayuda o biblioterapia y también con un número breve de sesiones, impartidas en pocas ocasiones por psicólogos cualificados, y la mayoría de las veces, por enfermeros. Esto es motivo de controversia y preocupación en dicho país. Al mismo tiempo, dicha situación genera un debate productivo del cual surgen y surgirán cosas buenas. En mi caso, formado en dicho país, mi interés por lo psicodinámico ha ido creciendo gradualmente, y parte de mi sesgo viene de mi fuerte formación cognitivista. Por tanto, creo que es posible trazar dicha tradición en lo que sigue, y espero mostrar que lejos de la confrontación, la realidad es que la psicología clínica goza de salud y fuerza. De hecho, creo que si Ferenczi levantara la cabeza, estaría orgulloso del logro de la asistencia sanitaria pública (en su versión de salud mental) que de alguna manera, alcanza a personas de toda clase social y por supuesto también al ver que la técnica psicoanalítica se ha adaptado a la realidad social contemporánea. 
En este trabajo, quisiera reflexionar sobre la utilidad del uso de metáforas en la práctica clínica dándole un sentido y una base relacional, dentro de la psicoterapia intersubjetiva, focal y breve. El motivo es por su potencial efecto sumativo con las técnicas y teorías relacionales, en un contexto de gran demanda de los servicios y la presión para poder contribuir a la materialización de cambios substantivos en las vidas de los pacientes en el menor tiempo posible.

\section{La Metáfora en un Contexto Psicoanalítico Relacional}

Nemiah (1976) en su definición de psicoterapia breve indica que es posible introducir modificaciones técnicas que impliquen adaptaciones teóricas. Si la introducción de metáforas construidas por el terapeuta y después co-construidas con el paciente, supusieran una potencial adquisición técnica en el campo relacional, podrían entonces también requerir cierto soporte teórico, que al menos en este momento creo que puede proceder de fuentes cognitivas y también relacionales, como me gustaría presentar a través de este trabajo. Desde el punto de vista relacional, para mí el lugar de salida es el reconocer que los modelos de terapia focal y de tiempo limitado relacionales facilitan el vivir una experiencia terapéutica que suponga un re-aprendizaje. Creo que la metáfora terapéutica entronca con esta perspectiva. En adición a este elemento fundamental, creo que la metáfora puede ser de ayuda para visualizar el concepto de "foco" y sus componentes psicoanalíticos fundamentales (i.e. conflicto intrapsíquico, defensas, ansiedades, contextualización del conflicto en las relaciones presentes). La metáfora puede potencialmente ayudar al paciente a desarrollar una ilusión como zona intermedia entre realidad y fantasía, tal y como Winnicott expresa, pudiéndose construir metáforas adaptadas a los focos temáticos, estratégicos y de situación. Generar una metáfora puede implicar que el analista revele su propia dinámica inconsciente y dependiendo de cómo construya y presente la metáfora, puede que muestre como va a tratar de ayudar y vincularse con su paciente. Esto puede permitir una exploración reflexiva de su propia actividad y de la calidad de la relación que está intentando generar (Ávila-Espada, et. al. 2002). Por otra parte, Bellak recomienda que se explique de forma sencilla la distorsión de la experiencia actual del paciente, en términos de sus experiencias anteriores, potenciando así su capacidad de cambio. La metáfora puede ser un medio eficaz para materializar dicho proceso. Por último, Bastos et. al. (2004) indican que la teoría intersubjetiva propone una flexibilidad que permite al analista investigar el impacto de sus técnicas y estilo personal sobre la experiencia del paciente. Esto también supone mayor libertad para explorar nuevos modos de intervención. Creo que la coconstrucción de metáforas puede encajar bien con esta perspectiva, ya que el paciente 
puede mostrar una evolución en el manejo de las metáforas de principio a fin de la intervención, no habiendo obstáculo para estratégicamente trabajar tanto patologías de conflicto como estructuras deficitarias, tal y como Ávila-Espada (2009) las define.

\section{Lo Narrativo de la Metáfora}

Se puede entender que el pensamiento humano tiene un elemento narrativo crítico, puesto que el mismo requiere de un contexto donde se describen una serie de sucesos y una línea argumental desde donde partir y también llegar a unas conclusiones (Bruner, 1987). Por ejemplo, una persona puede pensar que es débil, tras experimentar un breve episodio psicótico y justificarlo al seleccionar de su repertorio interpersonal, todas las cosas que creía haber hecho de forma incorrecta. De este modo, dichos fallos, producto de una asumida debilidad mental, le llevan lógicamente a un colapso o situación crítica, que la persona ha explicado narrativamente. Autores interesados en trabajar con la construcción terapéutica de narrativas alternativas, proponen que las construcciones narrativas negativas proceden de la cultura donde vive la persona, y que, por tanto, la persona ha podido incorporar creencias, modos de pensar y actuar que existen en su mundo social, aunque no se niega que cognitivamente la persona pueda construir activamente dichas historias (Rhodes \& Jakes, 2009). Creo que, desde una perspectiva psicodinámica relacional, se puede asumir que la persona puede describir su mundo interpersonal basándose directamente en la experiencia propia, sin tener que acudir necesariamente a las historias de otros para construir la suya (sin ignorar el peso de la cultura de fondo, que la persona viva en cada caso, como bien es reconocido entre autores asociados a dicha perspectiva; i.e. Ávila-Espada, 2009). El psicoanálisis relacional hipotetiza que la construcción fenomenológica de las creencias ha de basarse en la experiencia intersubjetiva. Las teorías freudianas, sin embargo, hablan de una mente cerrada (i.e. generada por un cerebro pre-programado con instintos y necesidades), y las cognitivas y psicosociales, de mentes condicionadas por los determinantes exteriores (i.e. una tabula rasa que permite la diversidad cultural y antropológica que todos conocemos). A través de la vía relacional podemos entender las narrativas personales como una manifestación del pensamiento y emoción humanos con una vertiente interpersonal autogenerada.

Volviendo a la capacidad humana para describir su vida de forma narrada o representacional, las metáforas parecen reflejar el pensamiento humano de forma tan palpable, que algunos autores han indicado que, a través de ellas, se vislumbra la esencia de la actividad mental dada su estructura figurativa y simbólica (Lakoff \& Johnson, 1980). Sin embargo, estos autores de inclinación cognitiva, pasan por alto la existencia de metáforas donde lo 
interpersonal juega un papel crucial, y en lugar de ello, hacen mención exclusiva a las metáforas como construcciones lógicas que explican un mundo físico y finito (Gonçalvez \& Craine, 1990) o quizás reflejando implícitamente, lo que se viene a llamar el concepto de mente aislada (Storolow \& Atwood, 1992). Tanto si se tiene un mayor interés en las metáforas de un tipo $u$ otro, parece claro que dichos procesos parecen ser eslabones fundamentales que representan la experiencia vivida y forman una cadena conceptual y afectiva con que coser y zurcir el tejido mental. De este modo no es difícil entender que las disfunciones psicológicas deben estar caracterizadas por organizaciones conceptualesmetafóricas inconscientes. Algunos autores cognitivos han desarrollado su propios métodos de trabajar con dichas representaciones dándole un cariz más intrapersonal (Young, Klosko \& Weishaar, 2003) o más basado en el constructivismo social (Rhodes \& Jakes, 2009), pero en todo caso y a mi modo de ver, perdiendo de vista lo interpersonal e intersubjetivo.

Desde mi punto de vista, por tanto, me gustaría ofrecer una aproximación a las metáforas de modo que se pueda ofrecer al paciente la posibilidad de re-estructurar su mundo interpersonal ofreciendo posibilidades de organización y comprensión alternativas a las que el paciente pueda tener en el momento de consulta. Esto es, ayudar a redirigir el proceso de construcción figurativa o conceptual de lo relacional basándonos en la naturaleza interpersonal de sus dificultades, carencias y conflictos y adaptando las metáforas al nivel de comprensión que la persona pueda manejar. A mi modo de ver, el ofrecer una narración o metáfora al paciente, se está haciendo un ofrecimiento de una historia ficticia con la que coconstruir el presente y quizás re-construir o re-entender el pasado. Siguiendo a Aron (1996) paciente y terapeuta se influyen mutuamente, dando lugar a una exploración de una realidad conjunta y significados mutuos co-construidos (Velasco-Fraile, 2009). De este modo la metáfora se puede presentar como una aportación temática con la que trabajar significados intra e interpersonales.

\section{Los Sueños y las Metáforas}

Desde un punto de vista representacional, los sueños son metáforas que el paciente comparte con el analista. El inconsciente es invisible, y los sueños, nos pueden servir para revelarlo (Pereira, 2009). Freud (1900) reconoce que los sueños son pensamientos. El paciente parece intentar inyectar su propia lógica a lo que narra en el sueño. El analista puede ayudar al paciente trabajando con dichas metáforas y narraciones simbólicas de sus conflictos. Pero, ¿podría el psicólogo ayudar al paciente si compartiera metáforas creadas a tal efecto? ¿Una metáfora creada ad hoc, sería una expresión más declarativa del proceso relacional que tiene lugar en la vida real y en la terapia? 


\section{La Metáfora del Perro Fiel}

Siguiendo a Velasco-Fraile (2009) el modelo relacional considera que existe un potencial conflicto a resolver entre la persona y sus progenitores, un conflicto explorable interpersonal e intrapsíquicamente, el cual contiene temáticas de fidelidad. La fidelidad hacia los padres es por tanto, el eje actitudinal desde donde partirán las relaciones presentes y futuras del individuo. En la metáfora del perro, se describe a un animal de compañía, que, por no desarrollar suficientemente su propia individualidad, puede confundir fidelidad con lealtad. El perro fiel, volverá a su amo tras recibir una paliza o quizás a pesar de experimentar cierto grado de descuido o desdén. El perro desea el contacto físico y la presencia de su amo a toda costa. Esto significa que el terapeuta utiliza ese patrón animal para extrapolarlo y así normalizar y justificar la necesidad real de apego. El perro fiel, puede continuar siéndolo, a costa de su propia integridad y dignidad. De hecho, se puede sugerir que la fidelidad es una actitud que se despliega incondicionalmente, independientemente de lo que suceda. Allí estará el perro, al servicio de su amo, pase lo que pase. Tras esta narración, se puede preguntar al paciente, si esa actitud es beneficiosa para el perro. Después, se podría decir de manera complementaria, que la lealtad depende de las circunstancias. Si el perro fuera leal, podría elegir entre continuar con un amo abusivo o escapar. Se puede explorar la normalización del abandono de una relación abusiva o indigna, para buscar relaciones más equilibradas y basadas en el respeto mutuo. De esta manera se pueden identificar y reconducir relaciones que no ayudan al paciente, introduciendo una narrativa y dos conceptos interpersonales interconectados (fidelidad y lealtad).

\section{La Metáfora del Juego de Cartas}

Los juegos pueden interpretarse como una representación comprimida de la vida interpersonal. Por ejemplo, en el caso de los juegos de cartas, éstos contienen importantes elementos relacionales, como el hecho de que existen una serie de normas y de criterios para conducirse a través del juego. Al final se trata de entender que, en dichos juegos, se interpreta un papel, de modo que lo importante es el "jugar" más que el asumir el papel que nos toca como algo fijo e inamovible. En los juegos de cartas pueden haber aliados y enemigos, y se puede mentir. Por otro lado, también se puede jugar torpemente, enseñando las cartas mientras se juega, casi sin darnos cuenta. Si se hace algo así, se acaba perdiendo el sentido del juego y es probable que todos los demás jugadores tomen una ventaja innecesaria sobre el jugador que las muestre. De hecho, parte de la atracción de los juegos de cartas, es el precisamente desarrollar la habilidad de contener y utilizar la información 
disponible de manera ventajosa, crear cierto grado de tensión tolerable y excitación de modo que se mantenga una motivación que ayude a todos los participantes a concluir el juego satisfactoriamente. Cuando en las relaciones humanas se toman las acciones personales de modo literal, puede sentirse que cada acto o interacción suponga un enorme riesgo, y el "jugador" se inhiba o rehúse jugar (dejando pasar su turno). Existen muchas submetáforas derivadas y adaptables al paciente y su problemática específica, que ayuden a reflejar su "juego" interpersonal.

\section{La Metáfora del Gobierno}

Loewald (2000) definió la segunda tópica de Freud en términos relacionales. De hecho, en el psicoanálisis relacional, lo intrapsíquico es una expresión de lo experimentado interpersonalmente (Velasco-Fraile, 2009). Más específicamente, en la psicología vincular, lo intrapsíquico es entendido como producto y reflejo de lo intersubjetivo (Ávila-Espada, 2005). Por tanto, la metáfora del gobierno introduce la posibilidad de visualizar la vida psíquica personal como el resultado de las relaciones entre distintos aspectos de la psique. Suponiendo que el sistema ejecutivo central fuera un presidente del gobierno, y las necesidades de la persona se representaran por los distintos ministros/ministerios del gobierno, entonces el paciente puede reconocer y aprender a tratar sus problemas y necesidades de manera relacional. Se le puede sugerir al paciente que si adopta el papel de ejecutivo, estará físicamente rodeado por su gabinete y probablemente, como en toda vida de relación, siempre acabamos desarrollando preferencias. Dichas preferencias suponen hablar y escuchar, por ejemplo, al Ministerio del Miedo, casi siempre, dejando otros ministerios importantes, completamente ignorados. Es por ello, por lo que acostumbramos a otros ministerios a guardar silencio y dejar que sólo los preferidos "hablen" con nosotros. Esta metáfora puede utilizarse para co-construir o reconstruir el Self de modo relacional y facilitar luego, una mejor vida de relación.

\section{La Metáfora del Ajedrez}

Como se ha descrito anteriormente, los juegos representan muchos aspectos de la vida social e interpersonal. La metáfora del ajedrez puede ayudar a entender de forma parecida a la metáfora del juego de cartas, a aceptar las relaciones como oportunidades para aprender, conocery afrontar la vida interpersonal de modo más lúdico y tentativo, más como un descubrimiento y exploración que como un espacio de relaciones que se poseen, o de las cuales se puede ser expulsado o privado. El ajedrez permite al igual que la metáfora del 
gobierno, utilizar como lugar de simulación, el interior de la mente misma, donde se lucha con un enemigo ficticio. Si al final se comprende el juego, la persona puede darse cuenta de que, en su interior, desde el tablero hasta las normas, todo es parte integral de la actividad psíquica de la persona, fomentando así la integración de aspectos rechazados o poco asumidos. En cuanto al uso de la metáfora como un reflejo de la vida relacional real, se puede utilizar enfatizando por ejemplo el aspecto fundamental de las relaciones, que puede ser el aceptar que cada uno tiene objetivos genuinos que fuerzan a querer influenciar al otro, a través de unas normas establecidas de antemano. Dentro de ese mundo determinista es posible movilizarse y relacionarse de formas infinitas, pero es necesario conocer bien las normas y jugar mucho para poder desarrollar pericia y seguridad en el juego. Esto lógicamente es una referencia metafórica a la necesidad de experimentar las relaciones liberándonos de los esquemas disfuncionales que hemos adquirido en la infancia.

\section{La Metáfora del Explorador}

Nuestra vida puede ser concebida como un camino con un principio y un fin. Entre esos dos extremos hay un mundo de posibilidades y oportunidades. Es frecuente encontrar en la clínica a pacientes que interpretan su vida como una serie de condicionantes que les limitan o no les dejan actuar a su gusto. Esas creencias desarrolladas a través de la vida de relación, pueden generar frustración y distrés. La metáfora del explorador puede ayudar a reconocer la gran cantidad de oportunidades que existen para poder elegir, no sólo en el "aquí y ahora", pero también en el futuro. También dicha metáfora ayuda a reconocer la inmovilidad del pasado, pero también la posibilidad de re-interpretación de su significado. De hecho, se puede decir que el punto de partida del explorador no tiene porqué marcar el trayecto o el final del camino, aunque muchos lo crean así. Es posible utilizar pequeños ejemplos de experiencias reales de exploración en donde puede haber otros personajes implicados, reales o imaginarios, con los que el paciente tiene que interactuar virtualmente y mostrar su capacidad para decidir por donde quiere conducirse. Los errores y los aciertos son parte de la vida del explorador, y sentirse perdido es algo casi imposible de evitar, cosa que ayuda a normalizar la propia construcción narrativa de la persona, la cual puede contener por ejemplo, mucha autoinculpación, autoexigencias o perfeccionismo. Creando una historia visual dibujada, puede ayudar a desarrollar a reconocer el guión que la persona seguía y el que alternativamente puede empezar a seguir a partir de ahora. La incertidumbre puede convertirse en inspiración y curiosidad. El descubrimiento del mundo y del otro, y el autodescubrimiento se pueden visualizar más claramente. Muchas veces se asume que la 
persona conoce su vida, para descubrir que aspectos básicos como carecer de una dirección o un objetivo relacional está en la base de sus dificultades y sufrimientos.

\section{Otros Usos Terapéuticos de las Metáforas}

Las metáforas anteriormente descritas creo que también pueden tener uso en el trabajo de valoración de los parámetros descritos por Leopold Bellack (ver González de Rivera \& de las Cuevas, 1992), aunque su exploración requeriría un estudio exclusivamente dedicado a este asunto.

\section{REFERENCIAS}

Aron, L. (1996). A meeting of minds: mutuality in psychoanalysis. Hillsdale, NJ. Analytic Press.

Avila-Espada, A. et.al. (2002). Reflexiones sobre la potencialidad transformadora de un psicoanálisis relacional. Intersubjetivo, 2, 4, 155-92.

Avila-Espada, A. (2005). Al cambio psíquico se accede por la relación. Intersubjetivo, 2, 7, 195-220.

Ávila-Espada, A. (2009). La psicoterapia psicoanalítica relacional: conceptos fundamentales y perspectivas. Simposio Interpsiquis, $10^{\circ}$ Congreso Virtual de Psiquiatría: La perspectiva relacional en psicoterapia psicoanalítica. Coordina: Prof Dr Alejandro Ávila-Espada- no referencia 1683325549.

Bastos, A. et al. (2004). Escenas que conmueven: los pivotes del cambio. Intersubjetivo, 1, 6, 129-43.

Bruner, J. (1987). Life as a narrative, Social Research, 54, 1, 11-32.

Camino, G (2019a). Transdiagnostic metaphors in clinical practice (part I). Journal of Clinical Psychology and Psychiatry. 3, 1, 14-20.

Camino, G (2019b). Transdiagnostic metaphors in clinical practice (part II). Journal of Clinical Psychology and Psychiatry. 3, 2, 14-20.

Freud, S. (1900). La interpretación de los sueños. En Obras completas. Buenos Aires, Amorrortu Editores, vol 4-5.

Gonçalves, O. \& Craine, M. (1990). The use of metaphors in cognitive therapy, Journal of Cognitive Psychotherapy, 4, 2, 135-50.

González de Rivera, J.L. \& de las Cuevas, C. (1992). La evaluación psicodinámica de las funciones del yo. Psiquis, 13, 8, 287-324.

Loewald, H.W. (2000). The waning of the Oedipus complex: Introduction. Journal of Psychotherapy Practice and Research, 9, 4, 239-49. 
National Collaborating Centre for Mental Health (2019). Improving access to psychological therapies.https://www.england.nhs.uk/wp-content/uploads/2019/02/improving-access-to-psychologicaltherapies-manual.pdf

Nemiah, J.C. (1976). Introduction, en D.H. Malan, The Frontier of Brief Psychotherapy, NY, Plenum Press.

Pereira, F. (2009). Del "Unbewusste" al inconsciente relacional. Clinica e investigación Relacional, 3, $1,68-81$.

Rhodes, J. \& Jakes, S. (2009). Narrative CBT for Psychosis. Routledge, East Sussex, UK.

Velasco Fraile, R. (2009). ¿Qué es el Psicoanálisis Relacional?. Clínica e Investigación Relacional, 3 (1): 58-67.

Young, E., Klosko, J.S., \& Weishaar, M.E. (2003). Schema Therapy: A practitioner's Guide. New York: Guildford Press.

Original recibido con fecha: 17/6/2019 Revisado: 19/8/2019 Aceptado: 30/09/2019 